\title{
Comprehending underspecified meaning: resolving ambiguity by contextual and conceptual search
}

\author{
Yao-Ying Lai; Maria Mercedes Piñango (Yale University)
}

\begin{abstract}
We examine sentence comprehension under conditions of semantic underspecification - one syntactic structure that supports multiple mutually exclusive semantic representations. We tested aspectual verb (AspVs) sentences such as "The queen began the novel" with agentive and constitutive readings, under biasing and neutral contexts. Results show that (i) whereas in the neutral context both readings were accessible, in the biasing context the non-supported reading was suppressed; and (ii) sentences were rated as more acceptable when presented with biasing context. The pattern suggests that semantic ambiguity influences acceptability, and that comprehenders resolve ambiguity by contextual and conceptual search.
\end{abstract}

\section{Introduction}

This study investigates the comprehension of underspecified sentence meaning-sentences with one overt syntactic structure but multiple semantic readings. Sentences with aspectual verbs (AspVs) like (1a) "The queen began the novel" may receive an agentive reading, in which the subject takes an agent role performing some activity associated with the complement denotation: (1b) "The queen began reading/writing/etc. the novel." However, this is not the only reading available. Sentences like (2) "This image begins the genealogy of the kings of England" "reveal instead a constitutive reading, in which the subject denotation is a subpart of the complement denotation. In fact, AspV sentences like (1a) can engender both an agentive reading (1b) and a constitutive reading such as (3) "(The story about) the queen was the first subpart of the novel." The constitutive reading is actually preferred in a supporting context such as "This novel vividly depicts every member of the British royal family" for example. The possibility of multiple readings is captured by the Structured Individual analysis (Piñango \& Deo, 2015), according to which an animate subject denotation in AspV sentences can be interpreted agentively or not depending on the dimensions along which the structure of the complement denotation can be construed and the context of the utterance. Using a questionnaire, we examine comprehension of AspV sentences with animate-denoting subjects under biased and nonbiased contextual conditions. We hypothesize that comprehenders resolve AspV sentences underspecification by a directed search through explicit and implicit context cues. If no disambiguating contextual information is available, the search to obtain an interpretation is not fruitful, and the interpretation remains ambiguous. The increased effort of maintaining multiple readings limits comprehensibility, resulting in a lower acceptability judgment.

\section{Method}

\subsection{Materials}

Thirty sets of short passages were created, each consisting of a context sentence followed by a target sentence with either an AspV or a psychological verb (PsychV) as controls. Each AspV sentence was paired with 3 types of context: (a) agentive biasing (agentBiasing), in which an event was mentioned (e.g., looked at) to hint the agentive reading, (b) constitutive biasing (constBiasing), in which a structured entity was mentioned (e.g., collections) to hint the subpart-hood reading, (c) Neutral, which allows both the agentive and constitutive readings. On the other hand, each PsychV sentence was paired with 2 types of context ${ }^{2}$ : agentBiasing or Neutral, while the context sentences were the same as those used for the AspV sentences. An example set of the 5 conditions is provided in Table 1.

\footnotetext{
${ }^{1}$ This example is taken from the Contemporary Corpus of American English (COCA).

${ }^{2}$ PsychV sentences were not paired with the constBiasing context because they do not give rise to a constitutive reading.
} 
Table 1: A set of sample stimuli (context sentence + target sentence with either AspV or PsychV)

\begin{tabular}{|c|c|c|}
\hline Condition & Context Sentence & Target Sentence \\
\hline $\begin{array}{l}\text { agentBiasing } \\
\text { (AspV / PsychV) }\end{array}$ & $\begin{array}{l}\text { In the past, writers often looked at collections of literary } \\
\text { work for inspiration. }\end{array}$ & \multirow{3}{*}{$\begin{array}{l}\text { William Shakespeare } \\
\text { began }(\mathbf{A s p}) \text { / enjoyed }(\mathbf{P s y c h V}) \\
\text { the volume containing works of classic } \\
\text { Renaissance comedies. }\end{array}$} \\
\hline $\begin{array}{l}\text { constBiasing } \\
\text { (AspV only) }\end{array}$ & Larry owns many collections of Renaissance literature. & \\
\hline $\begin{array}{l}\text { Neutral } \\
\text { (AspV / PsychV) }\end{array}$ & $\begin{array}{l}\text { Well-known writers usually owned collections of literary } \\
\text { work to look at for reference. }\end{array}$ & \\
\hline
\end{tabular}

\subsection{Participants \& Procedures}

Thirty native speakers of American English were asked to (i-acceptability rating task) rate the acceptability of each passage from a 1 5 scale, in which $5=$ fully understandable, and (ii-sentence interpretation task) choose the plausible interpretation(s) for the target sentence in the given context.

\subsection{Predictions}

We predict that the AspV sentences, due to their semantic ambiguity, would receive lower acceptability rating scores than the control PsychV sentences. Also, the Neutral-context condition would be rated lower than the Biasing-context conditions, because comprehension is harder without biasing information that guides comprehenders to arrive at one specific interpretation. With respect to the sentence interpretation task, comprehenders should obtain the respective reading in the two Biasing conditions of AspV sentences (agentBiasing-AspV, constBiasing-AspV), while the Neutral-AspV condition should engender both readings.

\subsection{Data Analyses}

One participant was excluded because the responses were undifferentiated. The remaining twentynine participants' responses were analyzed by mixed-model analyses in the $\mathrm{R}$ statistical environment (Baayen, 2008; R Core Team). Regarding the acceptability rating task, two sets of analyses were performed to address two research questions. (i) To access the context effect, we examined the rating scores of the three AspV conditions (agentBiasing-AspV, constBiasing-AspV, Neutral-AspV). A model with context type as the fixed factor was contrasted against a base model without it. (ii) We accessed the effects of verb type, context type, and their interaction by examining the scores of the four conditions as a $2 \times 2$ design. To this end, we incorporated two fixed factors in the models, each having two levels: Verb Type (AspV/PsychV) x Context Type (Neutral/agentBiasing).

Regarding the sentence interpretation task, we calculated the frequency of each response type chosen by the participants, per condition. For each AspV condition with a distinct context type, we performed a logistic mixed-model analysis to examine the difference between the agentive vs. constitutive reading. A difference in reading type (agentive vs. constitutive) was accessed by contrasting a model with a fixed factor of reading type against a base model without it.

\section{Results}

\subsection{Results of the acceptability rating task}

All five conditions with AspV \& PsychV were rated above the acceptable threshold $(>3.5)$ in average. (i) The analysis for AspV sentences showed a significant effect of Context Type $\left(\chi^{2}(2)=16.24\right.$, $p<.001$ ), in the direction of Neutral < constBiasing < agentBiasing. Pairwise comparisons (corrected) indicated that the Neutral condition was rated significantly lower than the agentBiasing condition $(p<$ .001) [Figure 1, left]. (ii) The analysis crossing Verb Type (AspV/PsychV) and Context Type (agentBiasing/Neutral) revealed a main effect of Verb Type, such that AspV sentences were rated significantly lower than PsychV sentences $\left(\chi^{2}(1)=9.43, p=.002\right)$. There was also a main effect of Context Type, such that the Neutral context was rated significantly lower than the agentBiasing context $\left(\chi^{2}(1)=20.88, p<.001\right)$ [Figure 1]. No interaction was found. 
Figure 1: Results of the acceptability rating task (1 5, 5=fully understandable)

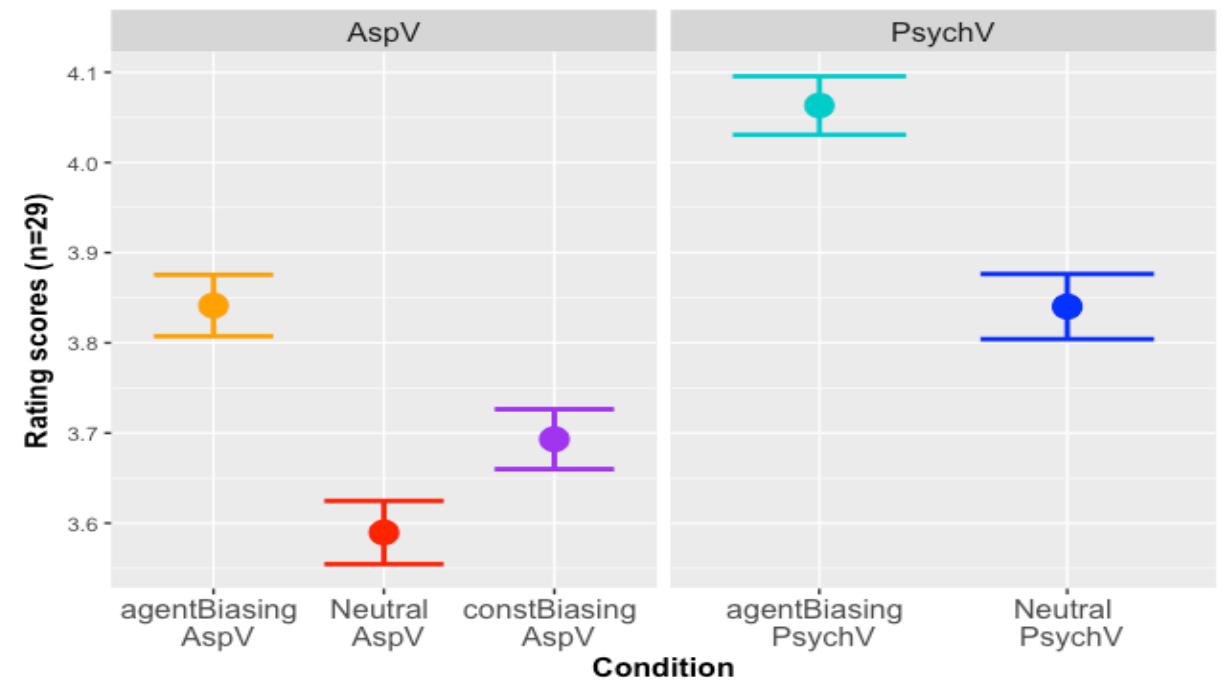

\subsection{Results of the sentence interpretation task-AspV sentences}

The AspV sentences in the Neutral context (Neutral-AspV) received the agentive and constitutive reading with roughly equal percentages - the ratio being [Agentive: Constitutive] $=1.19$. The two readings did not differ significantly $\left(\chi^{2}(1)=1.84, p=.175\right)$ for this condition. On the other hand, the agentBiasing context (agentBiasing-AspV) engendered the agentive reading twice more often than the constitutive reading; the two readings differed significantly $\left(\chi^{2}(1)=28.67, p<.001\right)$. In contrast, the constBiasing context (constBiasing-AspV) engendered the constitutive reading twice more often than the agentive reading; the two readings differed significantly $\left(\chi^{2}(1)=13.29, p<.001\right)$. [Figure 2]

Figure 2: Results of the sentence interpretation task-AspV sentences
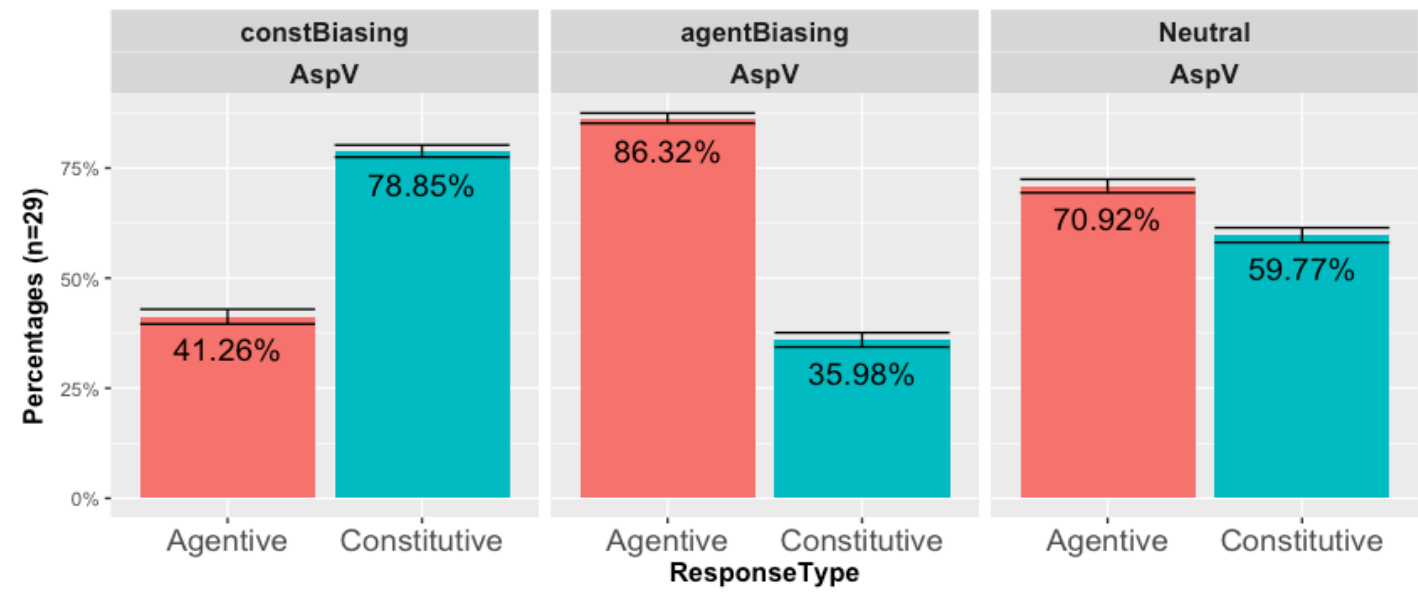

\section{Discussion \& Conclusion}

Results (Figure 2) show that in the neutral context AspV sentences engender an ambiguity between an agentive and a constitutive reading. In a biasing context, comprehenders settle on the supported reading at the expense of the alternative, thus indicating that the ultimate reading is contextually determined. In addition, AspV sentences which involve semantic ambiguity were deemed less acceptable than the PsychV counterparts. Also, ambiguous AspV sentences were deemed less acceptable in a 
nonbiasing context than in a biasing context (Figure 1).

The pattern suggests that sentence comprehensibility and acceptability are affected by the presence of semantic ambiguity and the quality of the context. In a neutral context, the presence of semantic ambiguity creates an uncertainty for comprehenders. The uncertainty and the additional effort of ambiguity maintenance limit comprehensibility. On the other hand, a biasing context resolves the underspecification, guiding comprehenders towards a specific reading. This increases comprehensibility and eases the processing effort which ultimately leads to greater acceptability. More generally, these results not only support one possible articulation of how an utterance's interpretation may ground itself in a context, but also suggest that sentence acceptability, a traditional proxy measure for grammaticality, may be impacted by processing factors, such as presence of ambiguity, beyond those strictly involving grammatical constraints.

\section{References}

Baayen, R. H. (2008). Analyzing linguistic data: A practical introduction to statistics using $R$. Cambridge University Press.

Piñango, M. M., \& Deo, A. (2015). Reanalyzing the complement coercion effect through a generalized lexical semantics for aspectual verbs. Journal of Semantics, 33(2), 359-408.

R Core Team (2015). R: A language and environment for statistical computing. R Foundation for Statistical Computing, Vienna, Austria. URL https://www.R-project.org/. 